\title{
Nonsense-mediated mRNA decay caused by a frameshift mutation in a large kindred of type 2 long QT syndrome
}

\author{
Ignatius Gerardo Zarraga, MD, * Li Zhang, MD, ${ }^{\dagger}$ Matthew R. Stump, PhD, * Qiuming Gong, MD, PhD, * \\ G. Michael Vincent, MD, ${ }^{\ddagger}$ Zhengfeng Zhou, MD, PhD* \\ From the *Division of Cardiovascular Medicine, Department of Medicine, Oregon Health \& Science University, Portland, \\ Oregon, ${ }^{\dagger}$ Main Line Health Heart Center, Jefferson Medical College, Philadelphia, Pennsylvania, and ${ }^{*}$ Department of \\ Medicine, LDS Hospital, University of Utah, Salt Lake City, Utah.
}

\begin{abstract}
BACKGROUND Nonsense and frameshift mutations are common in congenital long QT syndrome type 2 (LQT2). We previously demonstrated that hERG nonsense mutations cause degradation of mutant mRNA by nonsense-mediated mRNA decay (NMD) and are associated with mild clinical phenotypes. The impact of NMD on the expression of hERG frameshift mutations and their phenotypic severity is not clear.
\end{abstract}

OBJECTIVE The purpose of this study was to examine the role of NMD in the pathogenesis of a hERG frameshift mutation, P926AfsX14, identified in a large LQT2 kindred and characterize genotype-phenotype correlations.

METHODS Genetic screening was performed among family members. Phenotyping was performed by assessment of ECGs and LQTS-related cardiac events. The functional effect of P926Afs X14 was studied using hERG cDNA and minigene constructs expressed in HEK293 cells.

RESULTS Significant cardiac events occurred in carriers of the P926AfsX14 mutation. When expressed from cDNA, the P926AfsX14 mutant channel was only mildly defective. However, when expressed from a minigene, the P926AfsX14 mutation caused a significant reduction in mutant $m R N A$, protein, and hERG current. Inhibition of NMD by RNA interference knockdown of up-frameshift protein 1 partially restored expres- sion of mutant mRNA and protein and led to a significant increase in hERG current in the mutant cells. These results suggest that NMD is involved in the pathogenic mechanism of the P926AfsX14 mutation.

CONCLUSION Our findings suggest that the hERG frameshift mutation P926AfsX14 primarily results in degradation of mutant mRNA by the NMD pathway rather than production of truncated proteins. When combined with environmental triggers and genetic modifiers, LQT2 frameshift mutations associated with NMD can manifest with a severe clinical phenotype.

KEYWORDS Long QT syndrome; Mutation; Nonsense-mediated mRNA decay; Patch clamp; Potassium channel

ABBREVIATIONS hERG = human ether-a-go-go-related gene; $\mathbf{H P H}=$ hygromycin $\mathrm{B}$ phosphotransferase; $\mathbf{I}_{\mathbf{K r}}=$ rapid component of delayed rectifier potassium current; LQT2 = long QT syndrome type 2; LQTS = long QT syndrome; NMD = nonsense-mediated mRNA decay; NOS1AP = nitric oxide synthase 1 adaptor protein; PTC $=$ premature termination codon; shRNA = short hairpin RNA; SNP = single nucleotide polymorphism; UPF1 = up-frameshift protein 1 ; WT $=$ wild type

(Heart Rhythm 2011;8:1200-1206) ๑ 2011 Heart Rhythm Society. Published by Elsevier Inc. All rights reserved.

\section{Introduction}

The congenital long QT syndrome (LQTS) is a clinical disorder that affects close to 1:2,500 individuals in the general population. ${ }^{1}$ Its dramatic impact stems from its association with ventricular tachyarrhythmias and subsequent syncope or sudden cardiac arrest in otherwise healthy individuals. Several genetic variants of LQTS have now

This study was supported in part by National Institutes of Health (NIH) Grant HL68854 to Dr. Zhou and the Oregon Clinical and Translational Research Institute (OCTRI), Grant number UL1RR024140 01 from the National Center for Research Resources, a component of NIH, and NIH Roadmap for Medical Research. Dr. Zhou is an Established Investigator of the American Heart Association. Address reprint requests and correspondence: Dr. Zhengfeng Zhou, Division of Cardiovascular Medicine, CHH14Z, Oregon Health \& Science University, 3181 SW Sam Jackson Park Road, Portland, Oregon 97239. E-mail address: zhouzh@ohsu.edu. (Received January 12, 2011; accepted March 10, 2011.) been described, and, thus far, all are related to mutations in genes that encode cardiac ion channel subunits or proteins that modulate ionic currents. ${ }^{2,3}$ Long QT syndrome type 2 (LQT2) is the second most prevalent variant, accounting for $35 \%$ to $40 \%$ of genotyped cases of LQTS. ${ }^{2}$ It results from mutations in the human ether-a-go-go-related gene (hERG), also known as KCNH2. ${ }^{4}$ This gene encodes the $\alpha$-subunit of the channel that conducts the rapid component of the delayed rectifier potassium current $\left(\mathrm{I}_{\mathrm{Kr}}\right)$ in the heart. ${ }^{5-7}$

hERG mutations can cause channel dysfunction by a variety of mechanisms, including production of defective $\mathrm{I}_{\mathrm{Kr}}$ channels and defective protein trafficking. ${ }^{8,9}$ Recently, we showed that the nonsense-mediated mRNA decay (NMD) pathway is an important mechanism by which hERG nonsense mutations can influence the phenotypic severity of LQT2 ${ }^{10} \mathrm{NMD}$ is a surveillance mechanism that selectively degrades defective mRNA transcripts that con- 
tain premature termination codons (PTCs) resulting from nonsense or frameshift mutations. ${ }^{11,12}$ Notably, over $30 \%$ of the identified LQT2 mutations are nonsense or frameshift mutations that introduce PTCs. Although we previously demonstrated that NMD is a key player in determining the severity of disease in hERG nonsense mutations, the impact of NMD on the expression of hERG frameshift mutations and the phenotypic severity of LQT2 is not clear. ${ }^{10}$

In this study, we examined the role of NMD in the pathogenesis of a hERG frameshift mutation, P926AfsX14, which was identified in a large LQT2 kindred, and conducted genotype-phenotype correlation studies within the family. The P926AfsX14 mutation, which has also been referred to as G925fs/13, results from the insertion of guanine at nucleotide position 2775 of the hERG cDNA. The resulting alteration in the reading frame leads to the replacement of proline by an alanine residue in position 926 of the hERG protein and a PTC at position 14 in the shifted reading frame. A recent study by Nof et $\mathrm{al}^{13}$ indicated that hERG channels expressed from a hERG cDNA construct containing this mutation were only mildly defective. However, coexpression of P926AfsX14 with a common hERG polymorphism, K897T, led to a significant loss of hERG current, much greater than what was seen with expression of K897T or P926AfsX14 alone. Therefore it was concluded that the K897T polymorphism can markedly accentuate the loss of function of mildly defective hERG channels, and this mechanism was used to explain the LQTS-mediated arrhythmias and sudden infant death observed in that study. ${ }^{13}$ We, on the other hand, hypothesized that the P926AfsX14 mutation is subject to NMD, leading to degradation of the mutant mRNAs before they can produce partially functional hERG channels. This effect of NMD could, however, still lead to a severe clinical phenotype if it occurs in the presence of environmental triggers and genetic modifiers that increase the susceptibility to cardiac events.

\section{Methods \\ Clinical data}

The study was approved by the institutional review board and performed upon receipt of informed consent from subjects. The participants were blood-related members of a large family, many of whom had been previously identified as harboring the P926AfsX14 mutation. ${ }^{14}$ Phenotyping was performed based on the history of LQTSrelated cardiac events and assessment of QT intervals and T-wave morphology. ${ }^{15}$ Genotyping for the P926AfsX14 mutation, hERG K897T polymorphism, and nitric oxide synthase 1 adaptor protein (NOS1AP) polymorphisms was conducted by sequencing of genomic DNAs collected from blood or buccal swab samples.

\section{Plasmid constructs and transfection}

A minigene composed of hERG cDNA exons 1 to 10 and hERG genomic DNA from intron 10 to the poly(A) site was constructed by replacing the hERG cDNA C-terminal fragment with an intron-containing hERG genomic DNA frag-

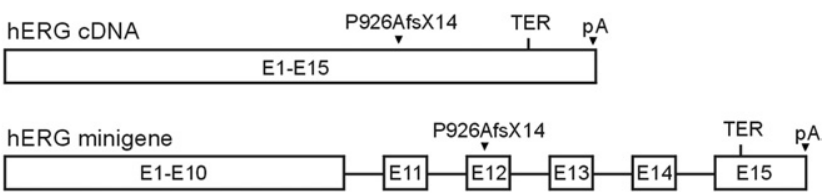

Figure 1 Diagram of the hERG cDNA and minigene structures. The positions of the wild-type termination codon (TER) and the P926AfsX14 mutation are indicated.

ment obtained from a human BAC clone, which consisted of the entire hERG gene (RP11-166D23) (Figure 1). The minigene was subcloned into a modified pcDNA5 vector in which the BGH poly(A) signal was deleted. Thus, the native poly(A) signal of the $h E R G$ gene was used for the formation of the poly(A) tail of hERG mRNA. The P926AfsX14 mutation was introduced into the hERG cDNA and minigene structures by site-directed mutagenesis using the pAlter in vitro mutagenesis system (Promega, Madison, WI, USA) (Figure 1). Flp-In HEK293 cells (Invitrogen, Carlsbad, CA, USA) were stably transfected with pcDNA5 hERG cDNA or minigene constructs and selected with 100 $\mu \mathrm{g} / \mathrm{mL}$ hygromycin B. Flp-In HEK293 cells contain the Flp recombination target sequence at a single genomic locus, allowing stable integration and expression of a single copy of the cDNA or minigene construct at a specific genomic location in all cell clones.

\section{RNase protection assay}

RNA isolation and the RNase protection assay were performed as previously described. ${ }^{10}$ Briefly, antisense RNA riboprobes were transcribed in vitro in the presence of biotin-14-CTP. The probe designed to detect the mRNA levels of the hERG minigene contained 278 nucleotides spanning the region of exons 13 and 14. The total length of the probe was 408 nucleotides and contained sequences from the pCRII vector at both ends. The probe for the hygromycin B resistance gene contained 158 nucleotides of the gene and 70 nucleotides from the pGEM vector. Yeast RNA was used as a control for the complete digestion of the probes by RNase. The relative intensity of each band was quantified using the Scion Image software (Scion Corp., Frederick, MD, USA).

\section{Immunoblot analysis}

Immunoblot analysis was performed as previously described. ${ }^{16,17}$ The cell lysates were subjected to SDS-polyacrylamide gel electrophoresis and then electrophoretically transferred onto nitrocellulose membranes. The membranes were probed with an anti-hERG antibody against the N-terminus of hERG protein (Santa Cruz Biotechnology, Inc., Santa Cruz, CA, USA). The expression level of hygromycin B phosphotransferase (HPH) encoded by the hygromycin B resistance gene in pcDNA5 vector was used as a loading control. ${ }^{18}$ A polyclonal anti-HPH antibody was used at a 1:1,000 dilution as previously described. ${ }^{19}$ The intensity of the protein bands was quantified using the Scion Image software. 


\section{RNA interference}

Up-frameshift protein 1 (UPF1) short hairpin RNA (shRNA) was used to inhibit expression of UPF1 as described previously. ${ }^{10,20}$ The shRNA targeted the coding sequence of hUPF1 (5'-GAGAATCGCCTACTTCACT-3'). HEK293 cells stably expressing wild-type (WT) or P926AfsX14 minigenes were stably transfected with plasmids containing the UPF1 or scrambled shRNA. The knockdown of the UPF1 protein was analyzed by immunoblot as previously described. ${ }^{10}$

\section{Patch clamp recordings}

Membrane currents were recorded in whole-cell configuration using suction pipettes as previously described. ${ }^{21} \mathrm{hERG}$ current was activated by a 4-second depolarizing pulse to 20 $\mathrm{mV}$ from a holding potential of $-80 \mathrm{mV}$. The hERG tail current was recorded following repolarization to $-50 \mathrm{mV}$. All patch clamp experiments were performed at room temperature $\left(22^{\circ}-23^{\circ} \mathrm{C}\right)$. Patch clamp data were presented as mean \pm SEM and analyzed by the Student's t-test. $P<.05$ was considered significant.

\section{Results}

\section{Phenotype and genotype analyses}

A total of 34 blood-related family members were included in the pedigree analysis after the proband was diagnosed with LQTS and was found to carry the P926AfsX14 mutation. The phenotype and genotype characteristics of each family member are shown in Figure 2. Twenty-two LQT2affected and nine unaffected individuals were identified based on the presence or absence of QT prolongation (with particular attention to bifid or notched $T$ waves) and/or history of LQTS-related cardiac events. The average QTc values for affected and unaffected individuals were $476 \pm$ $21 \mathrm{~ms}$ and $417 \pm 19 \mathrm{~ms}$, respectively $(P<.001)$. Nineteen affected and four unaffected individuals were available for genotyping. Consistent with the phenotype analysis, the 19 affected individuals were confirmed to be P926AfsX14 mutation carriers and the four unaffected individuals to be noncarriers.
Cardiac events were documented in nine mutation carriers. Seven had a history of recurrent syncope and two had cardiac arrest due to the use of QT-prolonging drugs or while under anesthesia during a hysterectomy. In addition, prior to the diagnosis of LQTS, three siblings of the proband had unexpected sudden death at ages 26, 28, and 32 years. Their genotypes are unknown, but one of them is an obligate mutation carrier, as her children were identified as mutation carriers. Sixteen individuals who were diagnosed with LQTS were subsequently placed on beta-blockers, and five received an implantable cardioverter-defibrillator.

A recent study suggested that a common hERG polymorphism, K897T, is an important genetic modifier for carriers of the P926AfsX14 mutation. ${ }^{13}$ Specifically, the presence of P926AfsX14 and K897T on separate alleles and their coexpression could lead to severe ventricular tachyarrhythmias and sudden infant death. To determine whether the K897T polymorphism might play a role in the phenotypic expression in the family in this study, we performed sequence analysis in eight mutation carriers (six with and two without a history of cardiac events). We did not find the K897T polymorphism in any of these individuals.

Variations in the gene for NOS1AP have also been reported to play a role in the development of cardiac events in LQTS patients. $^{22,23}$ Specifically, the presence of minor alleles in three NOS1AP single nucleotide polymorphisms (SNPs) rs4657139, rs16847548, and rs10494366 has been associated with an increased incidence of cardiac events in LQTS cohorts. To determine if these variant alleles might have played a role in the occurrence of cardiac events in this family, we performed an analysis of the three SNPs, rs4657139, rs16847548, and rs10494366, in eight mutation carriers (six with and two without a history of cardiac events). The two subjects who had no history of cardiac events did not carry variant alleles tagged by these SNPs, whereas four of the six mutations carriers who had a history of cardiac events carried these variant alleles. Of these four, three carried minor alleles in all three SNPs, and one carried minor alleles in two SNPs (rs4657139 and rs10494366).

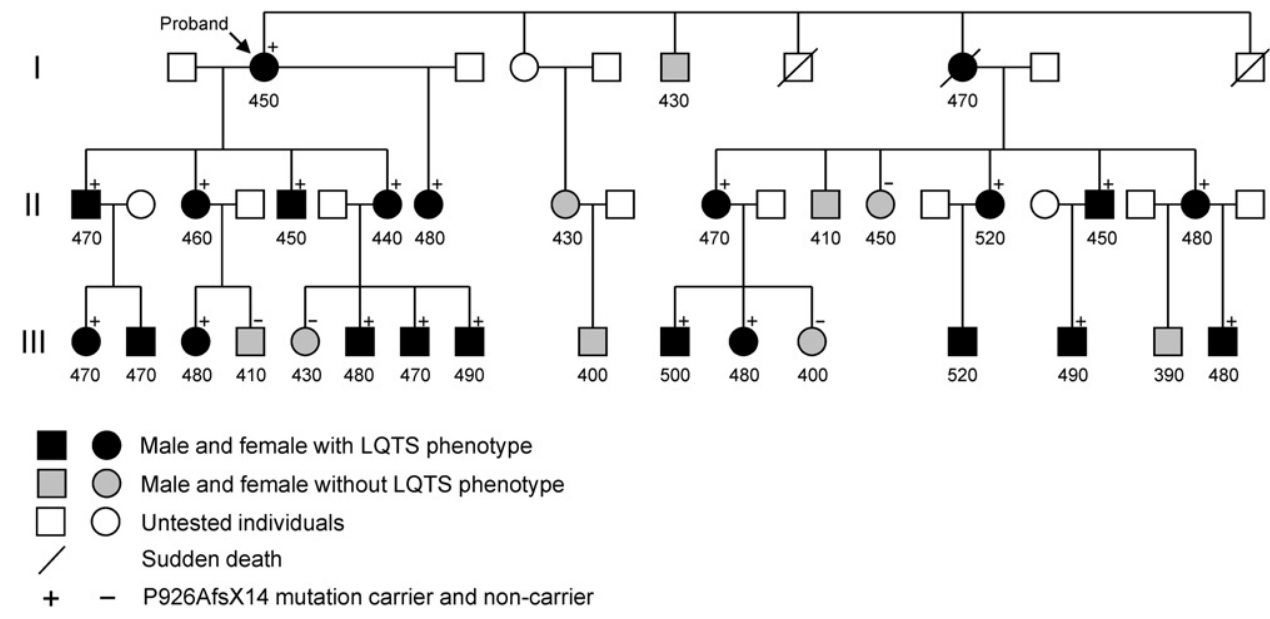

Figure 2 Pedigree of the family affected by the P926AfsX14 mutation. Numbers indicate corrected QT intervals. 


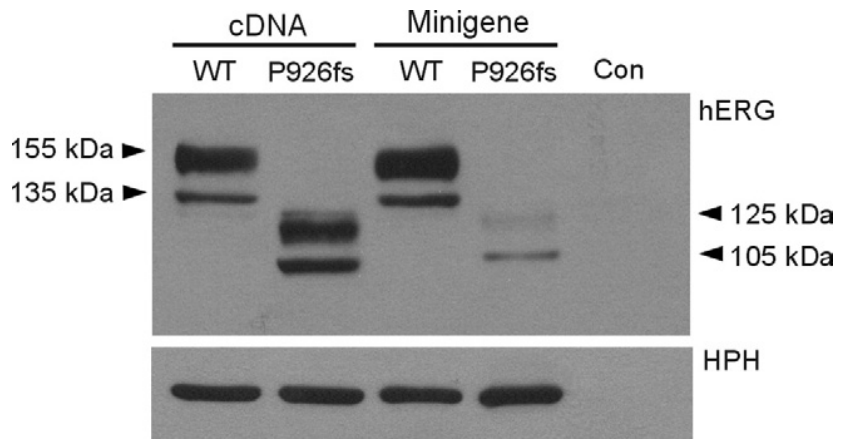

Figure 3 Western blot analysis of the hERG protein from HEK293 cells stably transfected with wild-type (WT) cDNA, P926AfsX14 cDNA, WT minigene, and P926AfsX14 minigene constructs. Cell lysates were subjected to SDS-PAGE and probed with anti-hERG and anti-hygromycin B phosphotransferase (HPH) antibodies. The level of HPH served as a loading control. Con $=$ untransfected control.

\section{Studies of the P926AfsX14 mutation using minigene and cDNA constructs}

To study the disease-causing mechanism of the P926AfsX14 mutation, we examined the mutation in the context of a minigene that contained hERG cDNA from exons 1 to 10 and hERG genomic DNA from intron 10 to the poly(A) site (Figure 1). When expressed in HEK293 cells, this minigene was properly spliced to produce the full-length hERG protein. The WT minigene expressed two protein bands at 155 and $135 \mathrm{kDa}$, consistent with the sizes of the hERG protein expressed from the hERG cDNA construct (Figure 3). As previously described, the $135-\mathrm{kDa}$ band represented the core-glycosylated immature form of the channel protein located in the endoplasmic reticulum, whereas the $155-\mathrm{kDa}$ band represented the complex-glycosylated mature form of the channel protein in the plasma membrane. ${ }^{8}$ The P926AfsX14 mutation, on the other hand, produced two smaller protein bands at about 125 and $105 \mathrm{kDa}$ (Figure 3). These bands corresponded to the mature $(125 \mathrm{kDa})$ and immature $(105 \mathrm{kDa})$ forms of the channel protein, with the smaller molecular weights reflecting the deletion of 221 residues due to the PTC at position 939 in the amino acid sequence. We observed significant differences in the levels of mutant proteins between cells transfected with hERG cDNA and the minigene. When expressed from cDNA constructs, the protein levels from WT and the P926AfsX14 mutant were comparable. In contrast, when expressed from the minigene, the level of the hERG protein from the P926AfsX14 mutant was markedly decreased compared to that seen from the WT minigene.

Functional analysis of the minigene-expressed hERG channels showed that the markedly reduced level of the P926AfsX14 protein resulted in a significant reduction in hERG current (Figure 4). The tail current amplitudes for the minigene-expressed WT and P926AfsX14 channels were $19.8 \pm 2.5 \mathrm{pA} / \mathrm{pF}(\mathrm{n}=8)$ and $1.5 \pm 0.2 \mathrm{pA} / \mathrm{pF}(\mathrm{n}=12$, $P<.05)$, respectively. We also analyzed the cDNA-expressed hERG channels and found that the P926AfsX14 mutation only caused a moderate reduction in hERG current. The tail current amplitudes generated by channels from WT and P926AfsX14 cDNAs were $19.2 \pm 1.7 \mathrm{pA} / \mathrm{pF}(\mathrm{n}=$ $8)$ and $11.4 \pm 1.0 \mathrm{pA} / \mathrm{pF}(\mathrm{n}=9, P<.05)$, respectively.

\section{Degradation of mutant mRNA by NMD}

To determine whether the reduction in protein expression and hERG current from the minigene-expressed P926AfsX14 mutant was due to degradation of mutant mRNA by NMD, we performed an RNase protection assay analysis using a probe spanning a region in exons 13 and 14 . The mRNA level from the P926AfsX14 minigene was found to be significantly lower than that from the WT minigene (Figure 5). Because degradation of mRNA by NMD is dependent on translation for nonsense codon recognition, inhibition of protein synthesis by a translation inhibitor such as cycloheximide would be expected to abrogate NMD of the mutant mRNA. The cells expressing the WT or P926AfsX14 minigene were therefore treated with cyclohexamide for 3 hours before RNA isolation. Treatment with cyclohexamide had no effect on the level of WT mRNA but significantly increased the level of P926AfsX14 mutant mRNA (Figure 5), suggesting that the P926AfsX14 mutant mRNA was degraded via the NMD pathway.
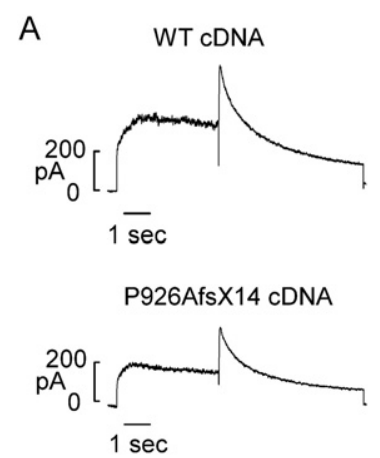
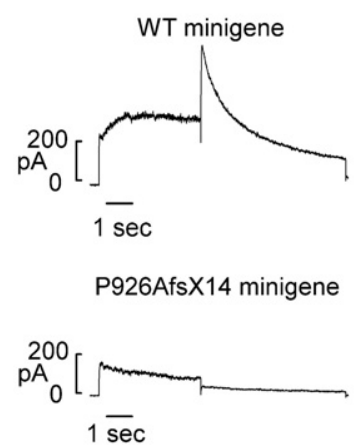

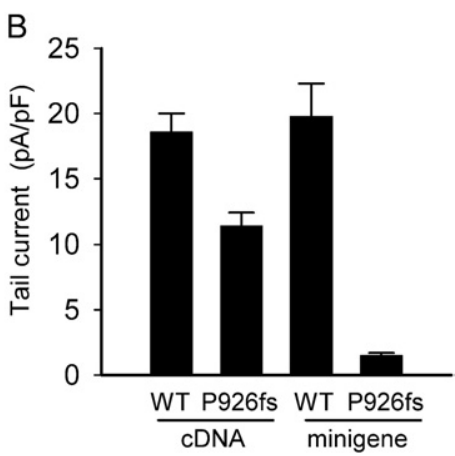

Figure 4 Patch clamp recordings of hERG current. A: Representative current traces from cells transfected with wild-type (WT) cDNA, P926AfsX14 cDNA, WT minigene, and P926AfsX14 minigene constructs as indicated. hERG current was activated by a 4-second depolarization pulse to $20 \mathrm{mV}$ from a holding potential of $-80 \mathrm{mV}$. hERG tail current was recorded by repolarization to $-50 \mathrm{mV}$. B: Histogram showing the average tail current density from the different cell groups. 

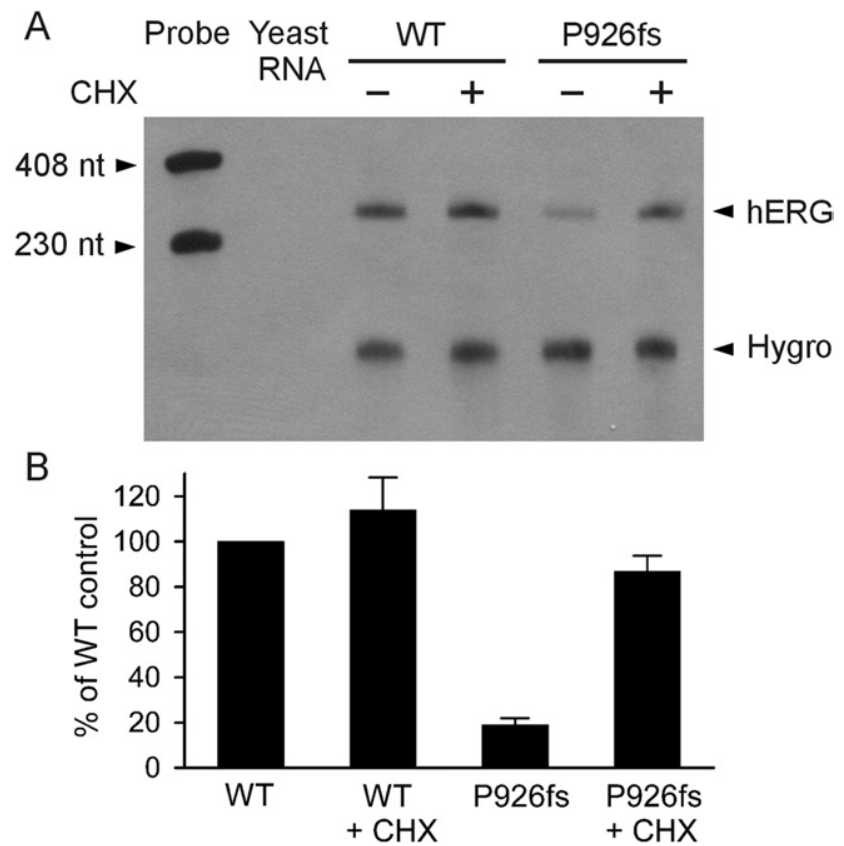

Figure 5 A: Analysis of hERG mRNA by RNase protection assay. HEK293 cells were stably transfected with wild-type (WT) or mutant hERG minigenes and subsequently treated $(+\mathrm{CHX})$ or not treated $(-\mathrm{CHX})$ with cyclohexamide for 3 hours prior to RNA isolation. The level of hygromycin B resistance gene transcript served as a loading control. B: Histogram showing the amount of hERG mRNA following normalization to hygromycin B resistance gene, plotted as a percentage of WT control from three independent experiments.

\section{Effect of UPF1 suppression on NMD of the P926AfsX14 mutation}

To further test the role of NMD in the reduction of protein expression and hERG current from the minigene-expressed P926AfsX14 mutant, we used the RNA interference method to knock down UPF1, which is a key protein in the core machinery of NMD. In these experiments, HEK293 cells

stably expressing WT and P926AfsX14 minigenes were transfected with UPF1 or scrambled shRNA plasmids. ${ }^{10}$ After using the RNA interference method, UPF1 knockdown in the cells was confirmed by Western blot analysis using anti-UPF1 antibody (Figure 6A). RNase protection assay analysis showed that the level of the mutant hERG mRNA was significantly increased in the UPF1 shRNAtransfected cells compared to that observed in scrambled shRNA-transfected cells (Figures 6B and 6C). The knockdown of UPF1 also resulted in an increase in the level of P926AfsX14 mutant protein (Figure 7). Patch clamp analysis revealed that UPF1 suppression significantly increased the hERG current amplitude in the mutant cells (Figure 8). The tail current amplitudes for scrambled shRNA-transfected cells and UPF1-suppressed cells were $1.3 \pm 0.2$ $\mathrm{pA} / \mathrm{pF}(\mathrm{n}=8)$ and $6.4 \pm 1.0 \mathrm{pA} / \mathrm{pF}(\mathrm{n}=9, P<.05)$, respectively. These results indicated that NMD was responsible for the degradation of P926AfsX14 hERG mRNA in the mutant cells.

\section{Discussion}

The findings of this study indicate that the NMD pathway plays an important role in the pathogenesis of the hERG frameshift mutation P926AfsX14. As an RNA surveillance mechanism, NMD operates by selectively degrading mRNA transcripts that contain PTCs from nonsense and frameshift mutations. ${ }^{11,12}$ Specifically, when translation terminates more than 50 to 55 nucleotides upstream of the $3^{\prime}$-most exon-exon junction of the mRNA, the mRNA is considered defective and is subjected to NMD. ${ }^{24}$ Because NMD depends on the occurrence of RNA splicing, the use of cDNA constructs, which bypasses RNA splicing, prevents PTCcontaining mRNA from being degraded by NMD. Therefore, experiments based on cDNA constructs may not always reflect the complete pathogenic mechanism of PTCcontaining mutations. In fact, when we used a hERG cDNA
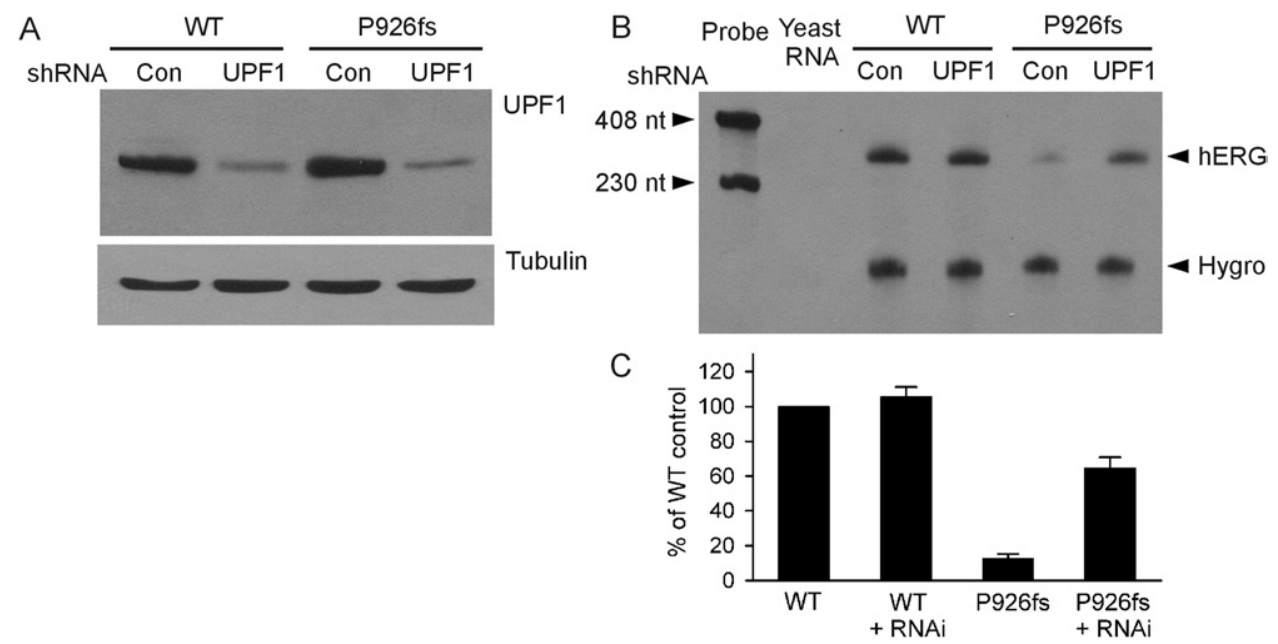

Figure 6 Effect of up-frameshift protein 1 (UPF1) suppression by RNA interference on hERG mRNA levels. HEK293 cells stably expressing wild-type (WT) or P926AfsX14 minigenes were transfected with either scrambled short hairpin RNA (shRNA) (Con) or UPF1 shRNA (UPF1). A: Western blot analysis of UPF1 protein. B: Analysis of hERG mRNA levels by RNase protection assay. C: Histogram showing amount of hERG mRNA following normalization to hygromycin B resistance gene, plotted as a percentage of WT control from three independent experiments. 


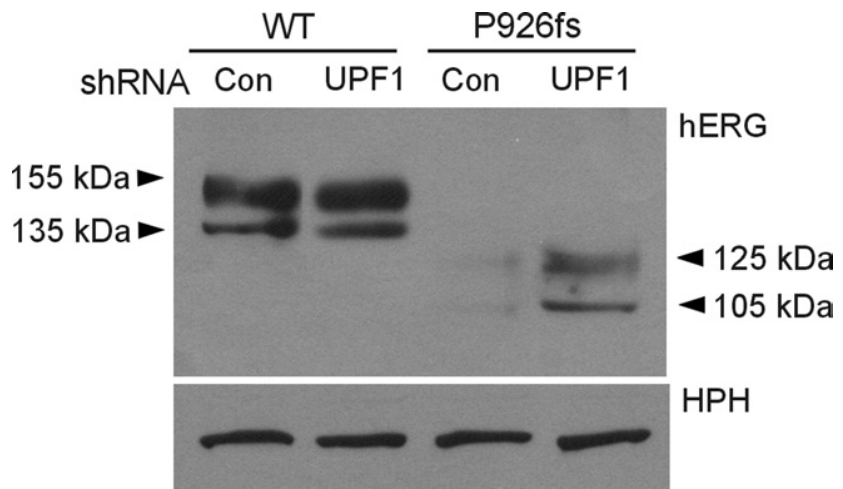

Figure 7 Effect of up-frameshift protein 1 (UPF1) suppression by RNA interference on hERG protein levels. HEK293 cells stably expressing wild-type (WT) or mutant hERG minigenes were transfected with either scrambled short hairpin (shRNA) (Con) or UPF1 shRNA (UPF1). Cell lysates were subjected to SDS-PAGE and probed with anti-hERG and antihygromycin B phosphotransferase (HPH) antibodies. HPH served as a loading control.

construct, the P926AfsX14 mutation only resulted in a moderate reduction in hERG current amplitude. In contrast, when we used a minigene construct that could generate the full-length hERG mRNA and protein and, importantly, could undergo RNA splicing, the same mutation caused a marked reduction in mutant protein levels and hERG current, a finding consistent with degradation of mutant mRNA by NMD. Therefore, our results underscore the importance of using minigene constructs when determining the effects of hERG PTC-containing mutations.

It was recently demonstrated that among patients with hERG mutations in the C-terminal region, those with nonmissense mutations, including PTC-containing mutations, had a significantly higher risk of arrhythmia-related cardiac events than did those with missense mutations. ${ }^{25}$ In some families with C-terminus mutations, we also demonstrated that NMD can be an important modifier of disease severity. Specifically, in LQT2 families that harbored the NMDsensitive R1014X and Q1070X mutations, individuals who were heterozygous for the mutation had a relatively benign cardiac phenotype. This was because NMD converted what would have been a dominant-negative effect from truncated proteins to haploinsufficiency by degrading mutant mRNAs before they could produce large amounts of defective protein. In contrast, in individuals who were homozygous for the same mutation, NMD led to a nearly complete elimination of hERG channels and a very severe cardiac phenotype. ${ }^{26}$ In the family that we analyzed in the present study, cardiac events were documented in nine mutation carriers. Seven had a history of recurrent syncope, and two had cardiac arrest due to the use of a QT-prolonging drug or while under anesthesia during surgery. Furthermore, three family members had premature sudden death of uncertain cause, and one of them was an obligate carrier of the P926AfsX14 mutation. Thus, this family had a fairly high incidence of cardiac events.

The study by Nof et $\mathrm{al}^{13}$ suggested that the K897T polymorphism can accentuate the loss of function of the
P926AfsX14 mutation because coexpression of K897T with P926AfsX14 led to a significant loss of hERG current, much greater than what was observed with expression of K897T or P926AfsX14 alone. However, their experiments were conducted in the context of cDNA constructs, which have their limitations as described previously. Our minigenebased experiment, which showed that degradation of the P926AfsX14 mutant mRNA by NMD led to a nearly complete loss of hERG current, has its own implications. In a heterozygous individual, the mutant allele would be expected to hardly contribute to the generation of hERG channels, whereas the WT allele generated functional hERG channels, thereby resulting in haploinsufficiency. However, if the WT allele in the same individual happened to harbor the K897T polymorphism, the resulting hERG current will be reduced by about $25 \%$ to $40 \%$ compared to a WT allele without this polymorphism. ${ }^{13,27,28}$ Therefore, in a heterozygous individual who carried both the P926AfsX14 mutation and K897T polymorphism, one can expect not only a reduction in the amount of hERG channels due to the NMD of mutant mRNA but also a reduction in the hERG current from the hERG channels containing the K897T polymorphism. ${ }^{13,27,28}$ This leads to a significant reduction in the overall hERG current and ultimately can manifest as a malignant cardiac phenotype. In the family we studied, we were able to perform sequence analysis on six individuals who had cardiac events, including two who had cardiac arrest. None of them harbored the K897T polymorphism, indicating that this could not be responsible for most of the cardiac events observed in this family. Whether or not it had a role in the three remote and premature sudden deaths in this family remains unknown because the genotypes of these individuals were not available.

Other gene variants have been implicated as modifiers of disease in LQTS. ${ }^{29}$ As mentioned previously, certain variants of the NOS1AP gene have been associated with a greater probability of cardiac events in patients with LQTS. $^{22,23}$ In the current study, the NOS1AP risk alleles were identified in four of eight individuals with the P926AfsX14 mutation. All four individuals carrying the NOS1AP variants experienced cardiac events, including
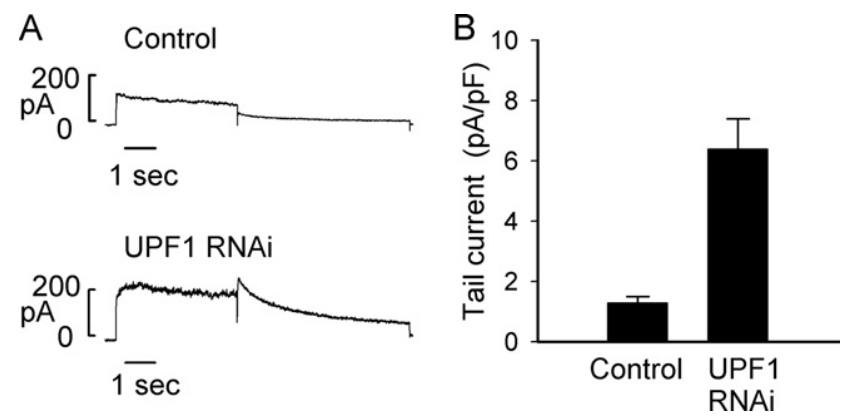

Figure 8 Effect of up-frameshift protein 1 (UPF1) suppression by RNA interference (RNAi) on hERG current. A: hERG current traces from cells stably expressing the mutant hERG minigene that were transfected with scrambled shRNA (Control) or UPF1 shRNA (UPF1 RNAi). B: Comparison of hERG tail currents measured from both cell groups. 
one cardiac arrest. Cardiac events were also observed in two individuals that did not carry the NOS1AP variants, one of whom experienced the cardiac event while under anesthesia during surgery. Our results suggest that the high incidence of cardiac events in this family might be partially explained by the coexistence of the NOS1AP risk alleles.

\section{Conclusion}

Our study showed that the P926AfsX14 frameshift mutation in hERG results in degradation of the mutant mRNA by the NMD pathway. When combined with environmental triggers (e.g., QT-prolonging drugs) and genetic modifiers that further increase the risk of cardiac events, the NMD mechanism can result in a severe clinical phenotype in patients carrying hERG frameshift mutations.

\section{Acknowledgments}

We thank the LQT2 family members for their cooperation and understanding of this research.

\section{References}

1. Schwartz PJ, Stramba-Badiale M, Crotti L, et al. Prevalence of the congenital long-QT syndrome. Circulation 2009;120:1761-1767.

2. Crotti L, Celano G, Dagradi F, Schwartz PJ. Congenital long QT syndrome. Orphanet J Rare Dis 2008;3:18.

3. Sanguinetti MC. HERG1 channelopathies. Pflugers Arch 2010;460:265-276.

4. Curran ME, Splawski I, Timothy KW, Vincent GM, Green ED, Keating MT. A molecular basis for cardiac arrhythmia: HERG mutations cause long QT syndrome. Cell 1995;80:795-803.

5. Sanguinetti MC, Jiang C, Curran ME, Keating MT. A mechanistic link between an inherited and an acquired cardiac arrhythmia: HERG encodes the $\mathrm{I}_{\mathrm{Kr}}$ potassium channel. Cell 1995;81:299-307.

6. Trudeau MC, Warmke JW, Ganetzky B, Robertson GA. HERG, a human inward rectifier in the voltage-gated potassium channel family. Science 1995;269:92-95.

7. Zhou Z, Gong Q, Ye B, et al. Properties of HERG channels stably expressed in HEK 293 cells studied at physiological temperature. Biophys J 1998;74:230241.

8. Zhou Z, Gong Q, Epstein ML, January CT. HERG channel dysfunction in human long QT syndrome. Intracellular transport and functional defects. J Biol Chem 1998;273:21061-21066.

9. Delisle BP, Anson BD, Rajamani S, January CT. Biology of cardiac arrhythmias: ion channel protein trafficking. Circ Res 2004;94:1418-1428.

10. Gong Q, Zhang L, Vincent GM, Horne BD, Zhou Z. Nonsense mutations in hERG cause a decrease in mutant mRNA transcripts by nonsense-mediated mRNA decay in human long-QT syndrome. Circulation 2007;116:17-24.
11. Conti E, Izaurralde E. Nonsense-mediated mRNA decay: molecular insights and mechanistic variations across species. Curr Opin Cell Biol 2005;17:316-325.

12. Kuzmiak HA, Maquat LE. Applying nonsense-mediated mRNA decay research to the clinic: progress and challenges. Trends Mol Med 2006;12:306-316.

13. Nof E, Cordeiro JM, Perez GJ, et al. A common single nucleotide polymorphism can exacerbate long-QT type 2 syndrome leading to sudden infant death. Circ Cardiovasc Genet 2010;3:199-206.

14. Splawski I, Shen J, Timothy KW, et al. Spectrum of mutations in long-QT syndrome genes. KVLQT1, HERG, SCN5A, KCNE1, and KCNE2. Circulation 2000;102:1178-1185.

15. Zhang L, Timothy KW, Vincent GM, et al. Spectrum of ST-T-wave patterns and repolarization parameters in congenital long-QT syndrome: ECG findings identify genotypes. Circulation 2000;102:2849-2855.

16. Gong Q, Jones MA, Zhou Z. Mechanisms of pharmacological rescue of trafficking-defective hERG mutant channels in human long QT syndrome. J Biol Chem 2006;281:4069-4074.

17. Gong Q, Zhang L, Moss AJ, et al. A splice site mutation in hERG leads to cryptic splicing in human long QT syndrome. J Mol Cell Cardiol 2008;44:502_ 509.

18. Gritz L, Davies J. Plasmid-encoded hygromycin B resistance: the sequence of hygromycin B phosphotransferase gene and its expression in Escherichia coli and Saccharomyces cerevisiae. Gene 1983;25:179-188.

19. Gong Q, Stump MR, Dunn AR, Deng V, Zhou Z. Alternative splicing and polyadenylation contribute to the generation of hERG1 C-terminal isoforms. J Biol Chem 2010;285:32233-32241.

20. Paillusson A, Hirschi N, Vallan C, Azzalin CM, Muhlemann O. A GFP-based reporter system to monitor nonsense-mediated mRNA decay. Nucleic Acids Res 2005;33:e54.

21. Gong Q, Anderson CL, January CT, Zhou Z. Pharmacological rescue of trafficking defective HERG channels formed by coassembly of wild-type and long QT mutant N470D subunits. Am J Physiol Heart Circ Physiol 2004;287:H652H658.

22. Crotti L, Monti MC, Insolia R, et al. NOS1AP is a genetic modifier of the long-QT syndrome. Circulation 2009;120:1657-1663.

23. Tomás M, Napolitano C, De Giuli L, et al. Polymorphisms in the NOS1AP gene modulate QT interval duration and risk of arrhythmias in the long QT syndrome. J Am Coll Cardiol 2010;55:2745-2752.

24. Nagy E, Maquat LE. A rule for termination-codon position within introncontaining genes: when nonsense affects RNA abundance. Trends Biochem Sci 1998;23:198-199.

25. Shimizu W, Moss AJ, Wilde AA, et al. Genotype-phenotype aspects of type 2 long QT syndrome. J Am Coll Cardiol 2009;54:2052-2062.

26. Bhuiyan ZA, Momenah TS, Gong Q, et al. Recurrent intrauterine fetal loss due to near absence of HERG: clinical and functional characterization of a homozygous nonsense HERG Q1070X mutation. Heart Rhythm 2008;5:553-561.

27. Anson BD, Ackerman MJ, Tester DJ, et al. Molecular and functional characterization of common polymorphisms in HERG $(\mathrm{KCNH} 2)$ potassium channels. Am J Physiol Heart Circ Physiol 2004;286:H2434-H2441.

28. Crotti L, Lundquist AL, Insolia R, et al. KCNH2-K897T is a genetic modifier of latent congenital long-QT syndrome. Circulation 2005;112:1251-1258.

29. George AL Jr. Common genetic variants in sudden cardiac death. Heart Rhythm 2009;6:S3-S9. 